\title{
Population Genetic Structure and the Migration of Puccinia striiformis f. sp. tritici Between the Gansu and Sichuan Basin Populations of China
}

\author{
Junmin Liang, Xiufeng Liu, Yong Li, Qiong Wan, Zhanhong Ma, and Yong Luo
}

\begin{abstract}
First, third, fourth, fifth, and sixth authors: Department of Plant Pathology, China Agricultural University, Beijing, 100193; and second author: Crop Research Institute, Tianjin Academy of Agricultural Sciences, Tianjin, 300081.

Current address of the first author: State Key Lab of Mycology, Institute of Microbiology, Chinese Academy of Sciences, Beijing, 100101. Current address of the fourth author: College of Plant Protection, Anhui Agricultural University, Hefei, Anhui Province, 230036. Current address of the sixth author: University of California, Kearney Agricultural Center, 9240 S. Riverbend Ave., Parlier 93648. Accepted for publication 17 October 2015.
\end{abstract}

\begin{abstract}
Liang, J., Liu, X., Li, Y., Wan, Q., Ma, Z., and Luo, Y. 2016. Population genetic structure and the migration of Puccinia striiformis f. sp. tritici between the Gansu and Sichuan Basin populations of China. Phytopathology 106:192-201

Puccinia striiformis f. sp. tritici is the causal pathogen of interregional epidemics of wheat stripe rust in China via long-distance migration. Gansu Province serves as putative inoculum center providing oversummering inoculum, while Sichuan Basin area serves as a region providing huge amounts of overwintering inoculum. Thus, the relationship between these two regions in population exchange and migration become important in prediction of interregional epidemics. In this study, we compared the population genetic structure and race composition between Gansu and Sichuan Basin populations to infer their migration relationships. A total

of 526 isolates, spanning 3 years, were genotyped using eight pairs of amplified fragment length polymorphism markers, and a subset of 98 isolates were inoculated onto 19 Chinese differentials to perform the race analysis. Twenty-three common races and 26 shared genotypes supplied molecular evidence for migration between Gansu and Sichuan Basin populations. Bayesian assignment and principal component analysis revealed that the genetic group assignment of the Sichuan Basin populations (10SB and 11SB) changed in the spring to align with the fall Gansu populations in the prior seasons (09GS and 10GS), which indicated an asymmetric migration from Gansu Province to the Sichuan Basin area. The linkage disequilibrium and the parsimony tree length permutation test revealed a strong annual recombination signal in the Gansu populations and an inconsistent signal in the Sichuan Basin populations.
\end{abstract}

Wheat stripe rust, caused by Puccinia striiformis f. sp. tritici, is an important wheat disease worldwide. Epidemics of the disease have occurred in northwestern Europe (Justesen et al. 2002), the United States (Chen et al. 1993), Australia and New Zealand (Steele et al. 2001), Pakistan (Bahri et al. 2011), the Mediterranean region (Bahri et al. 2009), and China (Wan et al. 2007). Compared with North America and Europe, China has a greater variation in geographical and climatic features, cultural characteristics (time of seed sowing and harvest, rotation, and irrigation), and varieties (Zeng and Luo 2006). Because China has the biggest epidemic region of wheat stripe rust in the world, the pathogen can affect over 20 million hectares of wheat via interregional epidemics (Zeng and Luo 2006). The annual yield loss due to stripe rust in China averages 1 million metric tons (Chen et al. 2007). During the most severe epidemics, occurring in 1950,1964, 1990, and 2002, yield losses of wheat approached $6.0,3.2,2.7$, and 1.4 million metric tons, respectively (Wan et al. 2002; 2007). A comprehensive understanding of the epidemiology of wheat stripe rust, population genetic structure, and interregional dispersal, especially in the oversummering and overwintering areas, is critical to design effective regional disease management strategies.

Based on research from the past five decades, 15 epidemic zones were classified in China, according to the unique features and the relationships among these regions in disease development and pathogen life cycle (Zeng and Luo 2006). Southern Gansu and eastern Gansu, where P. striiformis f. sp. tritici can both overwinter

Corresponding authors: Y. Luo; E-mail address: ygluo@ucanr.edu Z. Ma; E-mail address: mazh@cau.edu.cn

http://dx.doi.org/10.1094/PHYTO-03-15-0081-R

(C) 2016 The American Phytopathological Society and oversummer, were considered as the most important zones. The overlapping period (the "Green bridge") between sowing in the lowlands (late September) and harvesting in the highlands (early October) in these two zones could promote the survival of $P$. striiformis f. sp. tritici in its annual life cycle (Brown and Hovmøller 2002; Wan et al. 2004). Therefore, these two zones provide oversummering inoculum initiating interregional disease epidemics (Zeng and Luo 2006) due to the migration of urediniospores eastward to the regions where the pathogen cannot oversummer, with the potential of infecting autumn and spring plants.

With similar ecological characteristics, regions adjacent to Gansu Province are planted to wheat year around. These regions present complex climatic features. It is necessary to understand the migration pathways of the pathogen between Gansu and the adjacent provinces, so that the direction of inoculum movement and the target scope can be correctly estimated, and disease management is effective in the target regions after disease spread. Previous studies elaborated bidrectional dispersal varied with seasons (spring and fall) between Ningxia and Gansu populations (Liang et al. 2013) and main direction of the asymmetric dispersal was from Gansu to Qinghai populations (Lu et al. 2011). These results indicated various dispersal relationships of $P$. striiformis $\mathrm{f}$. sp. tritici populations between Gansu, as the source of $P$. striiformis f. sp. tritici inoculum, and its adjacent provinces.

Sichuan Province, located south of Gansu Province, covers the oversummering region of $P$. striiformis $\mathrm{f}$. sp. tritici in northwestern Sichuan (Fig. 1). The largest overwintering region is the Sichuan Basin area, where the pathogen can overwinter but not oversummer (Li and Zeng 2002) due to the heat. Because the pathogen cannot oversummer in Sichuan Basin, the source of the primary inoculum is unknown. One hypothesis is that the Sichuan Basin receives 
exogenous oversummering inoculum from source areas where $P$. striiformis f. sp. tritici could survive year-round, such as Gansu and northwestern Sichuan. Conversely, due to the warm winter in Sichuan Basin, wheat can continuously grow while $P$. striiformis $\mathrm{f}$. sp. tritici can overwinter with accumulated inoculum serving as a source of spring epidemics in other regions via spore dispersal by prevailing southeast wind in spring (Li and Zeng 2002). Moreover, Gansu and Northwestern Sichuan can provide year-round inoculum affecting interregional epidemics, since the pathogen can both oversummer and overwinter in these regions to accumulate inoculum (Zeng and Luo 2006). It is still questionable whether the overwintering inoculum from Sichuan Basin can migrate to Gansu.

The potential migration of $P$. striiformis $\mathrm{f}$. sp. tritici between Gansu and Sichuan Basin is a key issue to understand inoculum exchange over long distances in different seasons/years and in different regions. These factors could significantly affect interregional epidemics of stripe rust in China, but remain undetermined. A reasonable approach is to study the pathogen genetic structure in seasonal or temporal populations using molecular markers to infer the potential migration pathways. The current study compared the population genetic structure between the 2009 Gansu fall population and the 2010 Sichuan Basin spring population under the prevailing southeasterly winds. In addition, the 2010 Gansu fall population and the 2011 Sichuan Basin spring population were collected to confirm the migration direction revealed in the primary comparison.
Migration not only changes population genetic structure but also brings virulence alleles or new alleles to the target regions (McDonald and Linde 2002). Therefore, analysis of the dynamics of virulence genes can infer the possible migration route that occurred in the corresponding regions. For instance, pathotypes carrying virulence against the resistance gene $\operatorname{Yr} 9$ were used to track the migration route of $P$. striiformis $\mathrm{f}$. sp. tritici in several countries (Singh et al. 2004). In China, the race monitoring results have been used to predict possible epidemics of stripe rust. For example, four major pandemics of wheat stripe rust in China significantly related to the predominance of the Chinese yellow rust races CYR1, CYR19, CYR29, and CYR32, respectively, among the monitored populations (Wan et al. 2004; 2007). However, few studies implemented the correlation between race dynamics and migration among the pathogen populations in China. In this study, the race composition and frequency in Gansu and Sichuan Basin populations were studied to infer the possible migration pathway between these two important regions.

The objectives of the current study were to (i) compare the race composition of $P$. striiformis $\mathrm{f}$. sp. tritici in the Gansu and Sichuan Basin populations, (ii) determine the population genetic structure of $P$. striiformis f. sp. tritici in Gansu Province and the Sichuan Basin area, (iii) infer the major migration direction of $P$. striiformis f. sp. tritici between the Gansu and Sichuan Basin populations, and (iv) test the possibility of recombination in P. striiformis f. sp. tritici populations of Gansu and the Sichuan Basin.



Fig. 1. Sampling locations in Gansu and Sichuan Basin of China where Puccinia striiformis f. sp. tritici isolates were collected for this study. Samplings were conducted in different years (2009 to 2011). Regions with black color, dark gray color, and light gray color represent the zones where $P$. striiformis f. sp. tritici can both oversummer and overwinter, only oversummer, and only overwinter, respectively (Shi 2004). 


\section{MATERIALS AND METHODS}

Sampling and multiplication. To investigate the migration of P. striiformis f. sp. tritici between the Gansu and Sichuan Basin populations, 104 and 202 samples of $P$. striiformis f. sp. tritici were collected from Gansu Province in fall 2009 and 2010. These populations were coded as 09GS and 10GS. In addition, 110 and 110 samples were collected from the Sichuan Basin area in spring 2010 and 2011 and were coded as 10SB and 11SB, respectively (Table 1). Isolates collected from Gansu Province were located in the areas where $P$. striiformis f. sp. tritici can both overwinter and oversummer, while isolates from the Sichuan Basin area were located in the overwintering area only (Fig. 1). Single-pustule isolates were obtained and multiplied through inoculation on the susceptible cultivar Mingxian 169, as described by Liang et al. (2013). The inoculated plants were incubated in environment-controlled chambers with a $16 \mathrm{~h}$ photoperiod at $17 / 14^{\circ} \mathrm{C}$ (day/night). Approximately 15 to 17 days after inoculation, the spores were harvested, placed in silica gel-filled desiccators at $4^{\circ} \mathrm{C}$ for 2 to 4 days, and stored in liquid nitrogen for DNA extraction.

Race and virulence analysis. A total of 98 isolates (51 isolates for the Gansu population and 47 isolates for the Sichuan Basin population) were selected randomly for race analysis (Table 2 ). The following 19 standard differentials of wheat cultivars in China were used to determine the races of $P$. striiformis f. sp. tritici: Trigo Eureka (Yr6), Fulhard (unknown resistance gene), Lutescenes 128 (unknown resistance gene), Mentana (unknown resistance gene), Virgilio (YrVir1, YrVir2), Abbondanza (unknown resistance gene), Early Piemium (unknown resistance gene), Funo ( $\mathrm{r} A+$ ), Dsnidh 1 (Yr3), Jubilejina 2 (YrJu1, YrJu2, YrJu3, and YrJu4), Fengchan3 (Yrl), Lovrin $13(Y r 9,+)$, Kangyin 655 (Yrl, YrKy1, and YrKy2), Suwon 11 ( $\mathrm{YrSu}$ ), Zhong 4 (unknown resistance gene), Lovirn 10 (Yr9), Hybrid 46 (Yr3b, Yr4b), Triticum spelta ablum (Yr5), and Guinong (unknown resistance gene) (Chen et al. 2009).
All differentials were planted in 10 -cm-diameter pots with 7 to 10 seeds and grown in a spore-proof greenhouse. Approximately 10 days later, the first leaf was completely extended and the seedlings were ready to be inoculated. A fresh urediniospore suspension from each isolate was sprayed on the leaves. Inoculated seedlings were transferred to a dew chamber at 10 to $12^{\circ} \mathrm{C}$ for $24 \mathrm{~h}$ in the dark, and then transferred to a greenhouse under the same temperature with light conditions as described above. To prevent cross contamination, the inoculated plants were maintained separately from each other in individual plastic chambers during the reproduction process. When typical symptoms were fully developed, usually 15 to 20 days after inoculation, the infection type (IT) was assessed. The P. striiformis f. sp. tritici races were determined using a 0 to 4 IT scale (Stubbs 1985 ) in which scores from 0 to 2 were considered avirulent and from 3 to 4 were considered virulent. The race determination for 98 isolates was conducted without repeat when the infection type showed consistent virulence spectrum.

AFLP genotyping. Genomic DNA was extracted from 5 to $10 \mathrm{mg}$ of spores using a modified CTAB protocol (Enjalbert et al. 2002). Fresh urediniospores of each isolate were placed in a tube containing five to seven glass beads ( $5 \mathrm{~mm}$ in diameter) and shaken in a Bead Breaker (VORTEX-GENIE 2, Scientific Industries) with 3,000 strokes/min for 10 min followed by DNA extraction (Enjalbert et al. 2002). The extracted DNA was quantified on agarose gel $(0.8 \%)$ and was stored at $-20^{\circ} \mathrm{C}$ for further use. The amplified fragment length polymorphism (AFLP) procedure was carried out as described previously by Liang et al. (2013). All isolates were amplified by Pst $\mathrm{I}+0$ (5'-GTAGACTGCGTACATGCAG-3') and Mse I + 0 (5' GACGATGAGTCCTGAGTAA-3') primers first. The preselective production was diluted 30 times with $\mathrm{ddH}_{2} \mathrm{O}$ to make the template for selective amplification. The selective amplification was performed under the following conditions: 13 cycles of $94^{\circ} \mathrm{C}$ for $30 \mathrm{~s}, 65^{\circ} \mathrm{C}$ for $30 \mathrm{~s}$ with a $0.7^{\circ} \mathrm{C}$ decrement per cycle, $72^{\circ} \mathrm{C}$ for $60 \mathrm{~s}$, and then 25 cycles of $94^{\circ} \mathrm{C}$ for $30 \mathrm{~s}, 56^{\circ} \mathrm{C}$ for $30 \mathrm{~s}$, and $72^{\circ} \mathrm{C}$

TABLE 1. Summary information for Puccinia striiformis f. sp. tritici isolates collected from wheat fields in Gansu Province and the Sichuan Basin area, China

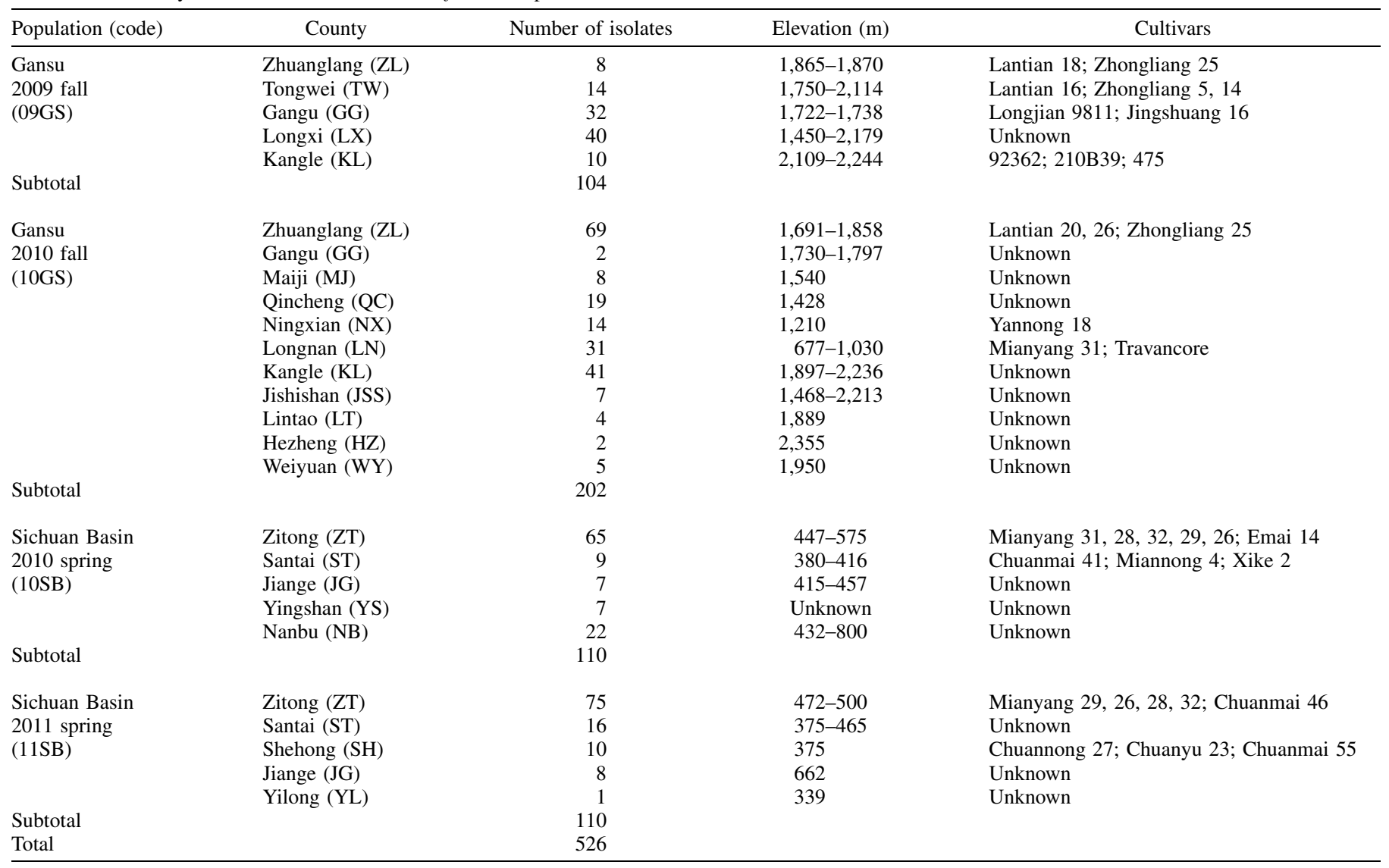


for $60 \mathrm{~s}$. Four Mse/Pst combinations [M12(AC)/P11(AA), M12 (AC)/P14(AT), M15(CA)/P16(CC) and M26(TT)/P12(AC)] were labeled with FAM fluorescent dye at $5^{\prime}$ end of the Mse primers and the other four primer combinations $[\mathrm{M} 25(\mathrm{TG}) / \mathrm{P} 15(\mathrm{CA}), \mathrm{M} 16$ (CC)/P12(AC), M17(CG)/P22(GT) and M16(CC)/P16(CC)] were also labeled at $5^{\prime}$ end of $M s e$ primers but with HEX fluorescent dye. Primer sequences were described previously (Vos et al. 1995). Selective amplification products were run in a denaturing polyacrylamide gel with an internal size standard (GS 500 ROX) on an automated DNA Sequencer (ABI 3730lx; Applied Biosystems, Carlsbad, CA). Replications were conducted for some isolates until the primary bands were consistent.

Genetic data analysis. Only informative polymorphic markers (presence or absence of a band shared by at least two isolates) were included in the AFLP data set. Polymorphic AFLP fragments were scored for each individual visually using GENOGRAPHER version 2.1.4 (Benham et al. 1999). Scores were assigned as 1 if the band was present and 0 if the band was not in a readable region ranging from 100 to $500 \mathrm{bp}$.

GenClone version 2.0 (Arnaud-Haond and Belkhir 2007) was used to check the suitability of the AFLP markers by plotting the number of multilocus genotypes detected against the number of loci chosen for genotypic diversity analysis. The parameter $P(\operatorname{sex})$ was estimated to test the possibility that repeated multilocus genotypes resulted from asexual reproduction. Within-population diversity was studied using gene diversity estimated by two indices, Nei's gene diversity $(H)$ (Nei 1972), and Shannon's information index $(I)$, using POPGENE version 1.3 (Yeh et al. 1997). MULTILOCUS version 1.3 (Agapow and Burt 2001) was used to calculate the number of distinct AFLP genotypes $(G)$ in four annual populations;
09GS, 10GS, 10SB, and 11SB. The genotypic diversity was calculated by dividing the number of distinct genotypes by the total number of isolates.

Pair-wise genetic differentiation of four annual populations, 09GS, 10GS, 10SB, and 11SB, were determined using the analysis of molecular variation (AMOVA) program in GENALEX version 6.4 (Peakall and Smouse 2006). $\Phi p t$, an analog of $F_{S T}$, was obtained for each pair-wise comparison with 1,000 permutations based on Nei's genetic distance matrices to estimate the genetic variation.

A Bayesian approach, performed in STRUCTURE version 2.4.1 (Pritchard et al. 2000), was used to group all multilocus genotypes to study genetic substructure in the Gansu and Sichuan Basin populations. All of $P$. striiformis f. sp. tritici isolates were assigned into $K$ clusters ranging from 1 to 10 based on their AFLP multilocus genotypes with clone correction. An admixture model and correlated alleles were used. Each $K$ was run for 10,000 iterations with a burn-in period of 1,000 , and 10 independent runs were repeated. Structure outputs were graphed as pie charts for each location based on the membership fraction (Q) Q $>0.8$. The best $K$ value was evaluated by $\Delta K$ as proposed by Evanno et al. (2005). Because the Bayesian approach is under the null model of panmixia, in which each locus is at Hardy-Weinberg equilibrium and is independent of the others (Gao et al. 2007), it may overestimate $K$ values if linkage occurs among some genotypes. Therefore, the genetic structure of $P$. striiformis f. sp. tritici populations in Gansu and the Sichuan Basin was also analyzed using a nonparametric approach, principal component analysis (PCA), which was performed using GENALEX version 6.4 (Peakall and Smouse 2006).

In order to study the direction of migration of $P$. striiformis f. sp. tritici between the Gansu and Sichuan Basin populations, two

TABLE 2. Race frequencies of Puccinia striiformis f. sp. tritici detected in Gansu and Sichuan Basin populations collected from wheat fields in this study

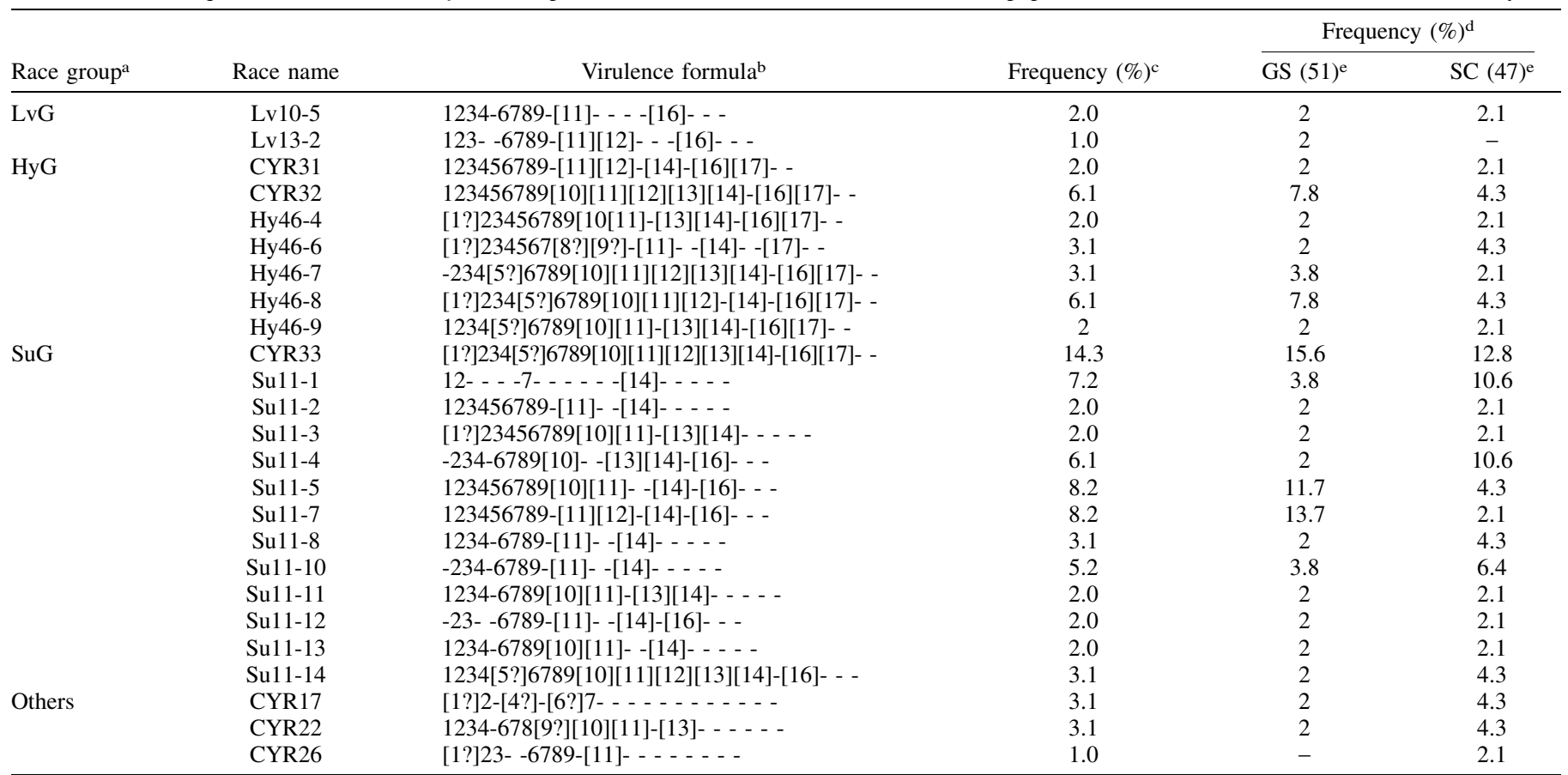

a $\mathrm{LvG}=$ race group virulent to Lovrin 10/Lovrin 13 possessing resistance gene of $Y r 9$; HyG = race group virulent to Hybrid 46 possessing resistance genes of $Y r 3 b$ and $\mathrm{Yr} 4 \mathrm{~b}$; and $\mathrm{SuG}=$ race group virulent to Suwon 11 possessing resistance gene of $\mathrm{YrSu}$.

b Numbers (1 to 19) represent 19 Chinese differentials: $1=$ Trigo Eureka $(Y r 6) ; 2=$ Fulhard (unknown); $3=$ Lutescenes 128 (unknown); $4=$ Mentana (unknown); 5 = Virgilio (YrVirl, YrVir2); 6 = Abbondanza (unknown); 7 = Early Piemium (unknown); 8 = Funo (YrA, +); 9 = Danish 1 (Yr3); $10=$ Jubilejina 2 (YrJu1, YrJu2, YrJu3, YrJu4); 11 = Fengchan $3(Y r 1) ; 12=$ Lovrin $13(Y r 9,+) ; 13=$ Kangyin $655(Y r 1, Y r K y 1, Y r K y 2) ; 14=$ Suwon $11(Y r S u) ; 15=Z$ Zhong 4 (unknown); $16=$ Lovrin 10 (Yr9); 17 = Hybrid 46 (Yr3a, Yr3b); 18 = Triticum spelta album (Yr5); and 19 = Guinong 22 (unknown). - represents infection types 0 to 2 and avirulence, and the numbers represent infection types 3 to 4 and virulence genes in corresponding differentials; ? indicates segregation of resistant and susceptible plants but more susceptible.

c Frequency of the corresponding race detected in all isolates.

${ }^{\mathrm{d}}$ Frequency of the corresponding race detected in each province.

e The values in parentheses represent the number of isolates used for race identification for each province. 
possible migration directions were represented using three temporal populations, 10SB, 10GS, and 11SB. Therefore, the existence of migrations from 10SB to 10GS indicated the direction from 2010 spring of Sichuan Basin to 2010 fall of Gansu, while that from 10GS to 11SB indicated the direction from 2010 fall of Gansu to 2011 spring of Sichuan Basin. Population subdivision, genetic group assignment, shared genotypes, and genetic differentiation were compared between populations in these two directions using the methods described above.

Recombination test. Two tests were conducted to examine the possibility of recombination in the Gansu and the Sichuan Basin populations, linkage of disequilibrium (LD) and the parsimony tree length permutation test (PTLPT) (Burt et al. 1996). Computations for PTLPT test were performed using PAUP version 4.0 (Swofford 1998) and statistical significance was assessed with 1,000 randomized data sets. To test the extent of departure from linkage equilibrium, two statistical measures, the index of association $\left(I_{\mathrm{A}}\right)$ and the proportion of phylogenetic compatibility (PrCP), were calculated using Multilocus 1.3 (Agapow and Burt 2001). The significance $(P)$ of $I_{\mathrm{A}}$ and $r_{\mathrm{d}}$ was tested by comparing the observed value with randomized data (1,000 permutations). The PrCP value approaching to 1 indicates no recombination (Agapow and Burt 2001; Chowdhary et al. 2011).

\section{RESULTS}

Twenty-five races were identified from 98 representative samples with race diversities of 0.47 in the Gansu population $(n=51)$ and 0.51 in the Sichuan Basin population $(n=47)$ (Table 2). Twentythree races, accounting for $92 \%$ of all identified races, were detected in both the Gansu and Sichuan Basin populations, with race frequency in each population (Table 2). The predominant races in these two populations were CYR33, Su11-5, Su11-7, Su11-1, Hy46-8, CYR32, Su11-4, and Su11-10, with total frequencies of $14.3,8.2,8.2,7.2,6.1,6.1,6.1$, and $5.2 \%$, respectively. The frequencies of other races were less than $5.0 \%$ (Table 2).

The eight pairs of AFLP primers generated 74 polymorphic fragments in the range of 100 to $500 \mathrm{bp}$ for all isolates. The number of polymorphic loci from each primer combination varied from 5 to 13. With the maximum loci limit defined in GenClone, only 38 loci were chosen randomly from 74 polymorphic loci to reveal the relationship between distinct genotype and number of loci used. Genotypic diversity was close to 1 at 28 loci (Fig. 2), meaning that $\geq 28$ polymorphic loci were meaningless to test genotypic diversity and caused the absence of shared genotypes. Finally, 11 loci with discrimination over $80 \%$ were selected randomly to analyze genotypic diversity and shared genotypes between populations. For other analyses, data sets of 74 polymorphic loci were still used. Based on 11 loci, a total of 457 distinct genotypes were identified. The Gansu population had a slightly higher genotypic diversity (0.93) compared with the Sichuan Basin population (0.91) (Table 3). Among 457 distinct genotypes, 419 were unique, and 38 genotypes were clones and were repeated two to four times.

Relationship among populations can also be determined by analyzing shared genotypes. Both six genotypes were shared between 09GS and 10SB, and between 10SB and 11SB; five and nine genotypes were shared between 10GS and 10SB, and 10GS and $11 \mathrm{SB}$, respectively (Fig. 3). The total of 26 genotypes provided direct evidences of migration between Gansu and the Sichuan Basin populations (Fig. 3).

The $P(\operatorname{sex})$ values of the 38 repeated genotypes were very low, ranging from $3.3 \times 10^{-6}$ for a single repeat to $2.3 \times 10^{-6}$ for four repeats. The low $P(\operatorname{sex})$ indicated that the resampled genotypes were not produced through recombination, but may have come from migration (Parks and Werth 1993). This serves as evidence for the possibility of migration between Gansu and Sichuan Basin populations.

The gene diversity estimated from Nei's $(H)$ and the Shannon index $(I)$ revealed that the Gansu $(0.482 \pm 0.023$ for $H$ and $0.675 \pm$ 0.023 for $I)$ and Sichuan Basin $(0.474 \pm 0.030$ for $H$ and $0.667 \pm$ 0.031 for $I$ ) populations had similar gene diversity (Table 3 ).

TABLE 3. Genotypic and genetic diversities of Puccinia striiformis f. sp. tritici in four temporal populations collected from wheat fields in Gansu Province and the Sichuan Basin area

\begin{tabular}{lcccc}
\hline Population & $n^{\mathrm{a}}$ & $G^{\mathrm{b}}$ & $H^{\mathrm{c}}$ & $I^{\mathrm{d}}$ \\
\hline 09GS & 104 & $98(0.94)$ & $0.406 \pm 0.104$ & $0.590 \pm 0.119$ \\
10GS & 202 & $193(0.96)$ & $0.487 \pm 0.020$ & $0.680 \pm 0.021$ \\
Combined & 306 & $285(0.93)$ & $0.482 \pm 0.023$ & $0.675 \pm 0.023$ \\
10SB & 110 & $102(0.93)$ & $0.420 \pm 0.059$ & $0.609 \pm 0.063$ \\
11SB & 110 & $104(0.95)$ & $0.477 \pm 0.031$ & $0.670 \pm 0.032$ \\
Combined & 220 & $200(0.91)$ & $0.474 \pm 0.030$ & $0.667 \pm 0.031$ \\
Total & 526 & $457(0.87)$ & $0.485 \pm 0.018$ & $0.678 \pm 0.019$ \\
\hline
\end{tabular}

a Sample size.

b Number of distinct genotypes based on 11 polymorphic loci (genotypic diversity $=$ number of distinct genotypes/all isolates in each subpopulation).

${ }^{c}$ Gene diversity $(H)$ within populations.

d Shannon index $(I)$ within populations.

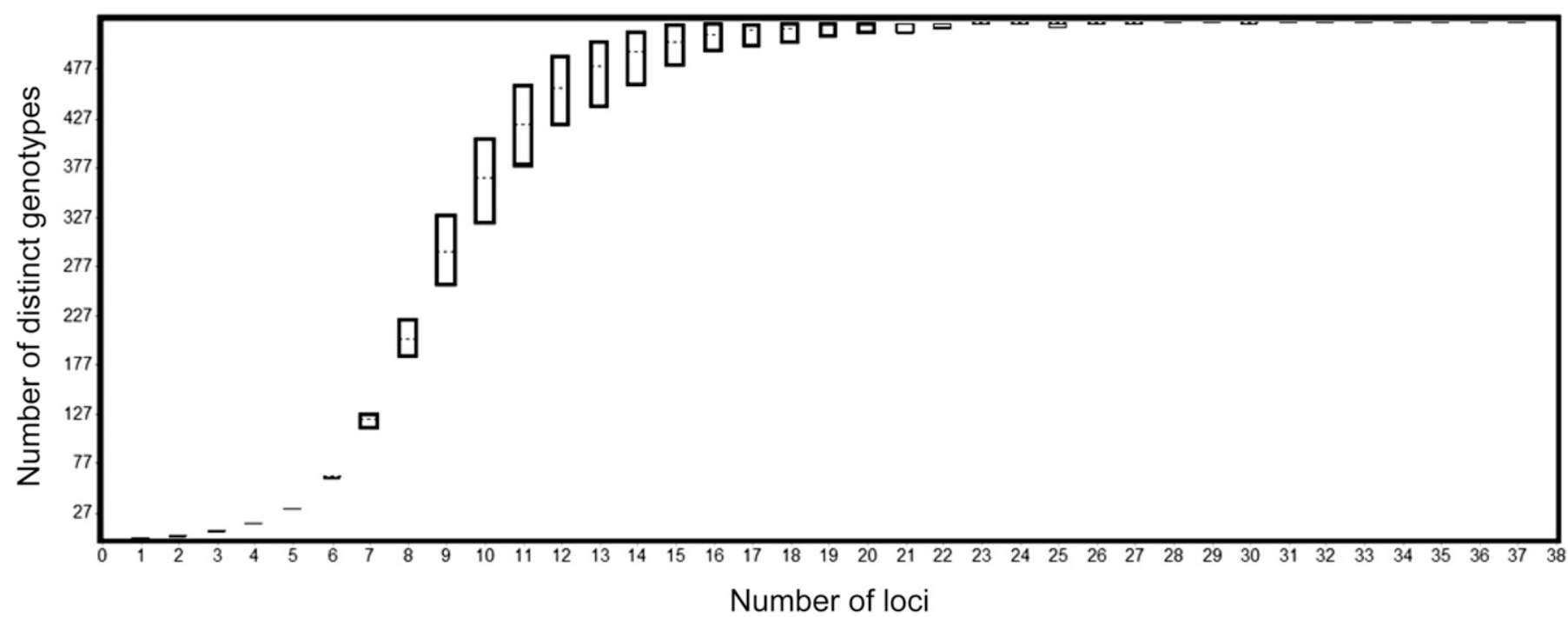

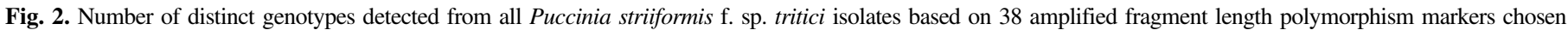
randomly from a total of 74 polymorphic loci using GenClone software. The bar represents the range of distinct genotypes detected when resampling on loci. 
However, between seasonal populations both in Gansu and Sichuan Basin, gene diversities were varied between seasonal populations in Gansu and Sichuan Basin populations and behaved as increasing in next seasons (10GS: $0.487 \pm 0.020$ for $H$ and $0.680 \pm$ 0.021 for $I$; $11 \mathrm{SB}: 0.477 \pm 0.031$ for $H$ and $0.670 \pm 0.032$ for $I$ ) compared with the prior seasons (09GS: $0.406 \pm 0.104$ for $H$ and $0.590 \pm 0.119$ for $I$; $10 \mathrm{SB}$ : $0.420 \pm 0.059$ for $H$ and $0.609 \pm 0.063$ for $I$ ) (Table 3).

The STRUCTURE output revealed that $\Delta K$ was maximized when $K=2(\ln K=-24816.8, \Delta K=295.4)$ based on the rate of change in the $\log$ probability of data across successive $K$ values (Evanno et al. 2005). At $K=2$, the genotypes obtained from 09GS (76.0\%) and 10SB (70.0\%) were mostly assigned into the black cluster $(\mathrm{Q}>0.5)$, while the genotypes obtained from 10GS (75.7\%) and 11SB (98.2\%) were mostly assigned into the white cluster (Q > 0.5) (Fig. 4A). The results showed a clear separation of the populations collected in 2009 to 2010 (09GS and 10SB) and 2010 to 2011 (10GS and 11SB). The genotypes assigned to the black cluster with a membership fraction $\mathrm{Q}>0.8$ were assigned to $\mathrm{G} 1$ group, those in the white cluster with $\mathrm{Q}>$ 0.8 were assigned to $\mathrm{G} 2$ group, and those with $0.2<\mathrm{Q}<0.8$ were considered as admixed genotypes and were assigned to admixed group (A group) (Table 4; Fig. 4B and C). In the Gansu populations (09GS and 10GS), $74.5 \%$ of the genotypes (228 genotypes) were admixed, significantly higher than in the Sichuan Basin populations $(10 \mathrm{SB}$ and $11 \mathrm{SB})\left(43.2 \%, P=9.629 \times 10^{-5}\right)$ (Table 4). Moreover, in the $09 \mathrm{GS}$ population, $24 \%$ of the genotypes were assigned to the G1 group, while only $5.8 \%$ of the genotypes were assigned to the G2 group. Whereas, many more genotypes (22.8\% of total genotypes) in the 10GS population were assigned to the G2 group compared with those assigned to the G1 group (only $0.5 \%$ of total genotypes) (Table 4). Interestingly, the group assignment of 10SB matched well with that of 09GS, and the group assignment of 11SB matched that of 10GS (Table 4). Thus, the assignment patterns of $P$. striiformis $\mathrm{f}$. sp. tritici populations changed over 2 years in each region. The patterns of subdivision from PCA were consistent with the results based on STRUCTURE software. The data were separated into two populations: one group included $83.6 \%$ genotypes of 09GS and $72.7 \%$ genotypes of 10SB, located in the first and forth quartiles which were significant at $P=0.04$, more than the proportions of 10GS $(36.1 \%)$ and $11 \mathrm{SB}(10.0 \%)$, and the second group was composed of $63.9 \%$ genotypes from 10GS and $90.0 \%$ genotypes from $11 \mathrm{SB}$, in the second and third quartiles which were significant at $P=0.04$, more than those of 09GS (16.3\%) and 10SB (27.3\%) (Fig. 5).

The pattern of population subdivision between temporal populations described above was further confirmed by pair-wise estimates of genetic differentiation $(\varphi p t)$. Although pair-wise $\varphi p t$ values were all significant $(P=0.001)$, two of the lowest $\varphi p t$ values were exactly between $10 \mathrm{GS}$ and $11 \mathrm{SB}(\varphi p t=0.029)$ and between $09 \mathrm{GS}$ and 10SB $(\varphi p t=0.054)$ (Table 5), suggesting that 09GS and $10 \mathrm{SB}$, as well as 10GS and 11SB, possessed the least genetic differentiation and could be treated as the same population.

From linkage disequilibrium test, the observed $I_{\mathrm{A}}$ and $r_{\mathrm{d}}$ values for $09 \mathrm{GS}, 10 \mathrm{GS}$, and $11 \mathrm{SB}$ except $10 \mathrm{SB}$ did not differ significantly from those calculated using randomly recombined data sets (Table 4). The PrCP values, all less than 1 in four temporal populations, indicated evidence of phylogenetic incompatibility, suggesting the recombination within populations (Table 4). From PTLPT analysis, the observed parsimony tree lengths of 09GS, 10GS and 11SB were 571, 805 , and 701 steps, respectively, located within the ranges of 1,000 randomized tree-length data sets at $P>0.05(P=0.100$ for 09GS, $P=0.210$ for $10 \mathrm{GS}$, and $P=0.105$ for $11 \mathrm{SB}$ ) (Table 4$)$. However, the tree length of 10SB (520 steps) was significantly $(P=0.001)$ shorter than those generated from 1,000 randomized data sets, suggesting that 10SB is a clonal population. Combined with all tests above, 09GS, 10GS, and 11SB showed strong signature of recombination while $10 \mathrm{SB}$ was predominantly clonal.

\section{DISCUSSION}

Our results showed that 26 AFLP genotypes and 23 races were shared between Gansu and Sichuan Basin populations and that migration occurred between them. Additionally, the primary direction of migration was inferred as from the Gansu population to the Sichuan Basin population, supported by evidence from analyses of shared genotypes, Bayesian assignment, and PCA analysis. These results confirmed our hypothesis that Gansu Province was the origin of extraneous inocula for epidemics in the Sichuan Basin area. Thus, the amount of inoculum in the Gansu fall population might be valuable data for estimating the risk of occurrence of stripe rust in spring in the Sichuan Basin area. More importantly, the amount of such inoculum can be critical to predict interregional epidemics of stripe rust because the occurrence of stripe rust in the Sichuan Basin

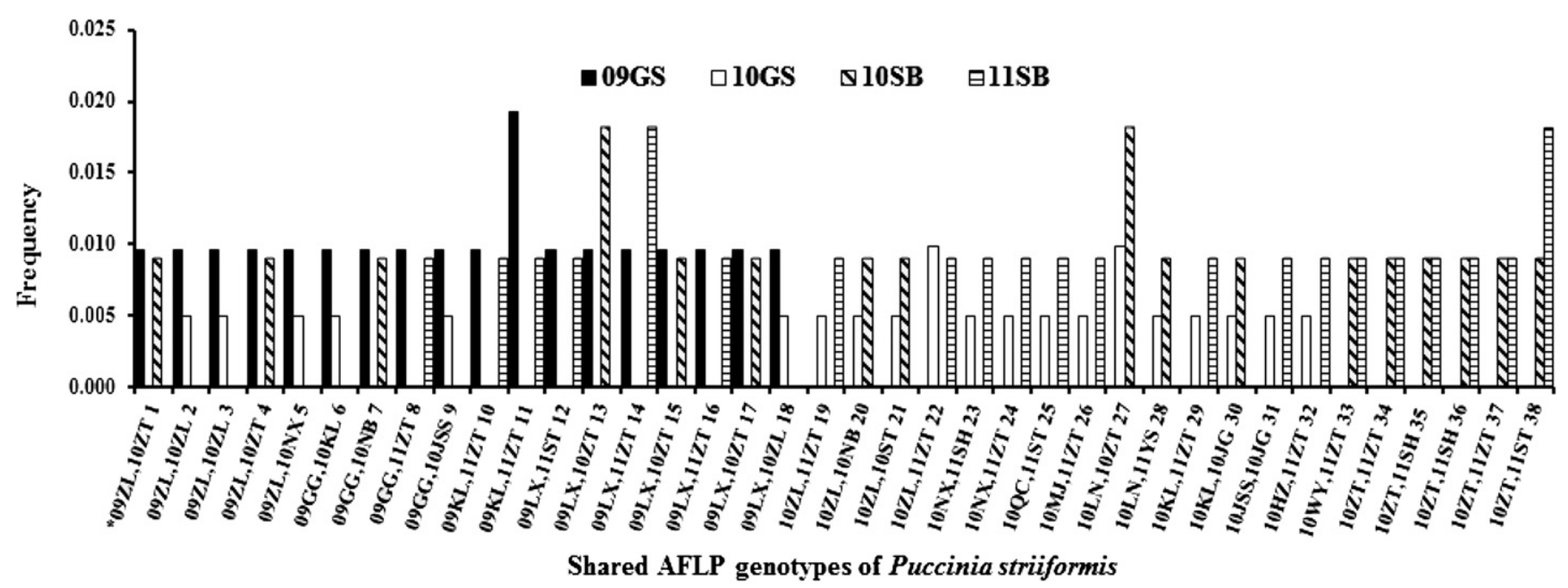

Fig. 3. Shared amplified fragment length polymorphism (AFLP) genotypes of Puccinia striiformis $\mathrm{f}$. sp. tritici in four temporal populations collected from Gansu and the Sichuan Basin. 09GS and 10GS = populations collected from Gansu in fall 2009 and 2010; 10SB and 11SB = populations collected from Sichuan Basin in spring 2010 and 2011. Shared genotypes were numbered sequentially from 1 to 38 . The geographic location of every shared genotype is labeled on horizontal axis as ZL = Zhuanglang County, Gansu; ZT = Zitong County, Sichuan; NX = Ning County, Gansu; GG = Gangu County, Gansu; NB = Nanbu County, Sichuan; JSS = Jishishan County, Gansu; KL = Kangle County, Gansu; LX = Longxi County, Gansu; ST = Santai County, Sichuan; SH = Shehong County, Sichuan; QC = Qingcheng County, Gansu; MJ = Maiji County, Gansu; LN = Longnan City, Gansu; YS = Yingshan County, Sichuan; JG = Jiange County, Sichuan; HZ = Hezheng County, Sichuan; and WY = Weiyuan County, Gansu. 
in early spring plays an important role in providing inoculum dispersing eastward (Zeng and Luo 2006).

Additionally, mountainous northwestern Sichuan is also both an oversummering and overwintering area for $P$. striiformis $\mathrm{f}$. $\mathrm{sp}$. tritici and could be another origin area providing inoculum for Sichuan Basin epidemics. Because "year-round" wheat is found in northwestern Sichuan, the overlapping period of crops from August to September provides ideal conditions for $P$. striiformis f. sp. tritici to oversummer locally, similar to the "green bridge" in Gansu Province (Li and Zeng 2002; Xie et al. 1998). In addition, a global survey of $P$. striiformis f. sp. tritici in Sichuan Province revealed that northwestern Sichuan and the Sichuan Basin populations had similar genetic diversity, indicating the possibility of gene flow between them (Wang et al. 2010). Future studies should focus on

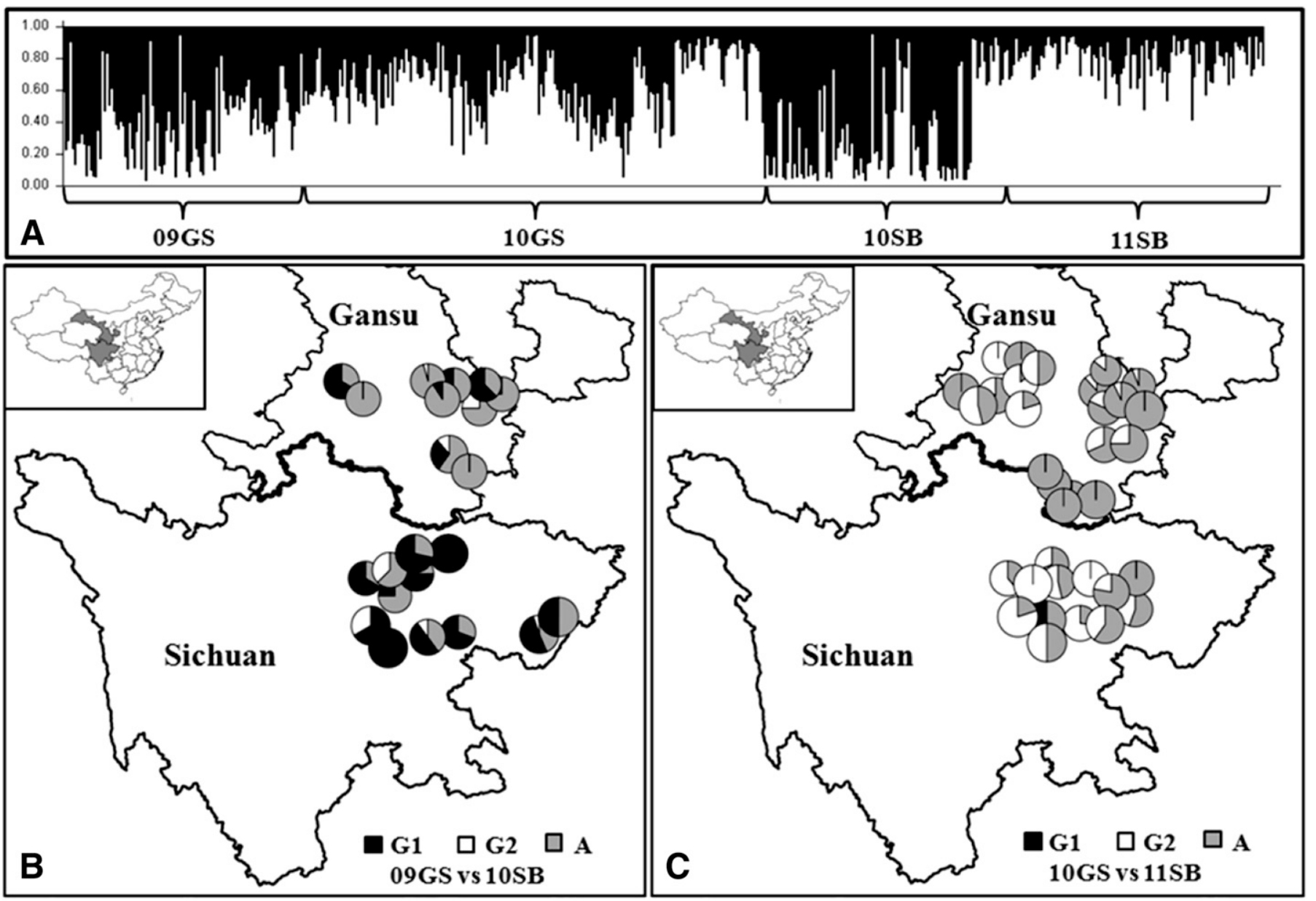

Fig. 4. Group assignment analysis of 526 Puccinia striiformis f. sp. tritici isolates from Gansu Province and the Sichuan Basin area. A, Assignment of isolates inferred from amplified fragment length polymorphism genotypes using STRUCTURE software. The horizontal axis consists of a single vertical bar for each isolate, broken into two groups (black and white). The vertical axis denotes estimated membership fraction (Q). B and C, Distribution maps of the two genotype groups in the four populations. G1 represents the isolates assigned into the black cluster in $\mathbf{A}$ with $\mathbf{Q}>0.8$. G2 represents the isolates assigned into the white cluster in A with $\mathrm{Q}>0.8$. A group represents admixed isolates from G1 and G2 groups.

TABLE 4. Bayesian assignments and recombination test of Puccinia striiformis f. sp. tritici for four temporal populations collected from Gansu Province and the Sichuan Basin area



a Group assignment based on membership fraction (Q) in STRUCTURE. G1 = group 1, including isolates assigned into G1 with Q $>0.8$, G2 = group 2, including isolates assigned into $\mathrm{G} 2$ with $\mathrm{Q}>0.8$ and $\mathrm{A}=$ admixed group, including admixed isolates with $0.2>\mathrm{Q}>0.8$.

b $I_{\mathrm{A}}$, index of association.

c $r_{\mathrm{d}}$, scaled $I_{\mathrm{A}}$ by the number of loci $(m-1)$.

d Percentage of phylogenetically compatible pairs (PrCP) of loci.

e Observed parsimony tree length for each temporal population.

f Ranges of parsimony tree lengths conducted from 1,000 randomized permutations using Mutilocus 1.3 (Agapow and Burt 2001). 
the contribution of extraneous inoculum from Northwestern China to early spring epidemics in the Sichuan Basin.

Several results in this study supported the inference of gene flow and the direction of migration from the Gansu population to the Sichuan Basin population, as follows. (i) Shared genotypes and common predominant races were detected between these two geographic locations, indicating that gene flow occurred between them. (ii) The low values of $P$ (sex) of all repeated/shared genotypes suggested that these genotypes were not descended from a recombination event but most likely from dispersal with gene flow between the Gansu and Sichuan Basin populations. (iii) More shared genotypes were detected between 10GS and 11SB (nine genotypes, $3.0 \%$ of total genotypes) than between 10SB and 10GS (five genotypes, $1.7 \%$ of total genotypes), suggesting more frequent gene flow from Gansu to the Sichuan Basin than in the reverse direction. (iv) The pair-wise $\varphi p t$ value between 10GS and 11SB was 0.029 , much lower than that between 10SB and 10GS $(\varphi p t=0.055)$ (Table 5). (v) Moreover, PCA showed that 10GS and 11SB could be considered as one population separate from 10SB, demonstrating a higher genetic identity between populations in the direction from Gansu to the Sichuan Basin. (vi) The components of the Bayesian assignment of two spring Sichuan Basin populations matched well with those of two Gansu fall populations in the previous season, implying a change of population structure in the Sichuan Basin area following that of Gansu population. This observation suggests that the genetic structure of the Sichuan Basin population might be affected by the Gansu population via migration. In summary, these results indicate migration between the Gansu and Sichuan Basin populations and demonstrate that the main migration direction was from Gansu (fall source) to the Sichuan Basin (spring target).

Usually, host selection would particularly favor the fittest virulent phenotypes/genotypes, thus increasing their frequencies (McDonald and Linde 2002). However, in spite of various major varieties carrying different resistance genes between Gansu and Sichuan Basin populations, 23 out of 25 total races were shared between them and the predominant race groups were both focused on the HyG and SuG groups (Table 2). For instance, the major wheat varieties of Zhongliang and Lantian in the Gansu population possess the resistance genes $\mathrm{Yr} 3, \mathrm{Yr} 3 a+, \mathrm{Yr} 4 a+, \mathrm{Yr} 16$, and $\mathrm{Yr} 9$ (Cao et al. 2011) compared with $Y r 2, Y r A$, and $Y r 15$ in the wheat varieties Mianyang and Chuanmai in the Sichuan Basin population (Xia et al. 2005; Yang and Stubbs 1990).
The frequency of the shared genotype is used to infer migration in many studies. One way is based on a time series of the same genotype. For instance, Hovmøller et al. (2008) reported the migration of two aggressive $P$. striiformis f. sp. tritici strains from south-eastern United States to Australia. The same genotype was detected at the source location 2 years earlier than the target location. Another way is to compare the frequencies of the shared genotype at the same time in both the source and target regions. The migration directions between Gansu and two adjacent provinces, Ningxia (Liang et al. 2013) and Qinghai provinces (Lu et al. 2011), were inferred in this way. In this study, we combined two ways to infer the possible migration direction between Gansu and Sichuan Basin populations. Our conclusion of migration from the Gansu population to the Sichuan Basin population is also supported by previous epidemiological evidences showing a time series of interregional development of the disease in China over different epidemic regions (Zeng and Luo 2006). To date, the important adjacent regions (Ningxia, Qinghai, Shaanxi, and Sichuan provinces) of Gansu Province were primarily studied and the integrated results confirmed the role of Gansu Province as the center of inoculum of P. striiformis f. sp. tritici for interregional epidemics of wheat stripe rust in China.

Population structure analysis served as a tool to reveal the migration from Gansu to the Sichuan Basin. First, the population structure as component proportions of Bayesian assignment was determined as G1, G2, and A groups in Gansu population in the fall of 2009 (09GS). The genotypes in the following population (10SB) were also assigned into three groups. Then the Bayesian structure changed in the Gansu population in the fall of 2010 (10GS) with genotypes assigned in two predominant groups ( $\mathrm{G} 2$ and $\mathrm{A})$ and the population structure of $11 \mathrm{SB}$ changed correspondingly into $\mathrm{G} 2$ and $\mathrm{A}$

TABLE 5. Estimates of $\Phi p t$ (lower diagonal) and its significance (upper diagonal) among four temporal populations of Puccinia striiformis $\mathrm{f}$. sp. tritici collected from wheat fields in Gansu Province and the Sichuan Basin area ${ }^{\mathrm{a}}$

\begin{tabular}{lllll}
\hline & 09GS & 10GS & 10SB & 11SB \\
\hline 09GS & $\ldots$ & 0.001 & 0.001 & 0.001 \\
10GS & 0.062 & $\ldots$ & 0.001 & 0.001 \\
10SB & 0.054 & 0.055 & $\ldots$ & 0.001 \\
$11 \mathrm{SB}$ & 0.120 & 0.029 & 0.123 & $\ldots$ \\
\hline
\end{tabular}

a $\Phi p t$, an analog of $F s t$, was calculated as the proportion of the variance among subpopulations using GENALEX 6.4 and its $P$ value was determined by 1,000 random permutations.

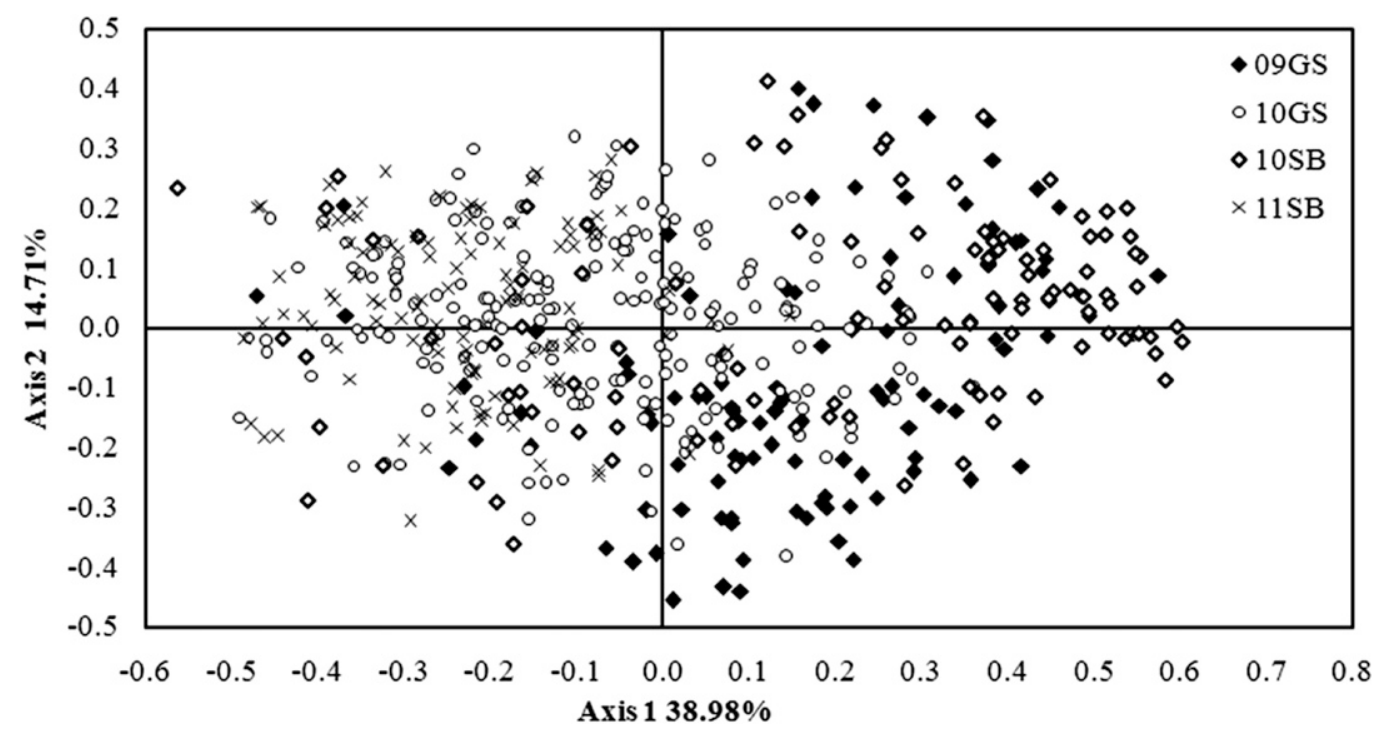

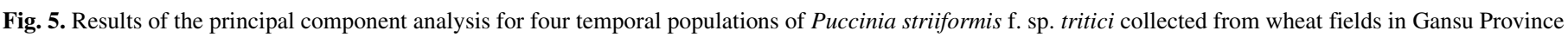


Basin in spring 2010 and 2011. 
groups (Table 4). Clearly, the change in population structure in Gansu followed that in the Sichuan Basin, giving a hint that the origin of inoculum in Sichuan Basin was from the Gansu fall population.

In addition, the low possibility of recombination in the temporal Sichuan Basin population reflected that the Sichuan Basin population may be also affected by spores dispersed from other regions such as Gansu (Liu et al. 2011; Mboup et al. 2009) where recombination clearly existed. This scenario may affect the population structure in the Sichuan Basin such that the two temporal populations showed a difference in recombination signal.

Combined with previous studies, the direction of migration between Gansu and its adjacent provinces, Qinghai (Lu et al. 2011), Ningxia (Liang et al. 2013), Shaanxi (Wang et al. 2010), and Sichuan Basin, can be inferred. However, the mutual relations among these adjacent provinces remain undetermined. With the development of an approximate Bayesian computation model and its successful application to $P$. striiformis f. sp. tritici to infer migration routes among worldwide populations (Ali et al. 2014), its utilization in determining migrations of $P$. striiformis f. sp. tritici populations in China has become a reality.

\section{ACKNOWLEDGMENTS}

We thank Y. Jin and J. Kolmer (USDA-ARS Cereal Disease Lab), and W. Boming (Department of Plant Pathology, China Agricultural University) for their valuable comments and suggestions on this manuscript prior to submission; L. Hui of the Gansu Plant Protection Station and S. Li of the Sichuan Plant Protection Station for providing P. striiformis f. sp. tritici samples; and L. Weifeng, Y. Minglu, W. Fei, and G. Yating (Department of Plant Pathology, China Agricultural University) for their assistance in reproduction of $P$. striiformis $\mathrm{f}$. sp. tritici isolates. This study was supported by the Natural Science Foundation of China (Project 31371881) and the National Key Basic Research Program of China (Project 2013CB127700).

\section{LITERATURE CITED}

Agapow, P. M., and Burt, A. 2001. Indices of multilocus linkage disequilibrium. Mol. Ecol. Notes 1:101-102.

Ali, S., Gladieux, P., Leconte, M., Gautier, A., Justesen, A. F., Hovmøller, M. S., Enjalbert, J., and de Vallavieille-Pope, C. 2014. Origin, migration routes and worldwide population genetic structure of the wheat yellow rust pathogen Puccinia striiformis f. sp. tritici. PLoS Pathog. 10:e1003903.

Arnaud-Haond, S., and Belkhir, K. 2007. GenClone: A computer program to analyze genotypic data, test for clonality and describe spatial clonal organization. Mol. Ecol. Notes 7:15-17.

Bahri, B., Leconte, M., Ouffroukh, A., de Vallavieille-Pope, C., and Enjalbert, J. 2009. Geographic limits of a clonal population of wheat yellow rust in the Mediterranean region. Mol. Ecol. 18:4165-4179.

Bahri, B., Shah, S. J. A., Hussain, S., Leconte, M., Enjalbert, J., and de Vallavieille-Pope, C. 2011. Genetic diversity of wheat yellow rust population in Pakistan and its relationship with host resistance. Plant Pathol. 60:649-660.

Benham, J., Jeung, J. U., Jasieniuk, M., Kanazin, V., and Blake, T. 1999. Genographer, a graphical tool for automated AFLP and microsatellite analysis. J. Agric. Genomics 4:15-19.

Brown, J. K. M., and Hovmøller, M. S. 2002. Aerial dispersal of pathogens on the global and continental scales and its impact on plant disease. Science 297:537-541.

Burt, A., Carter, D. A., Koenig, G. L., White, T. J., and Taylor, J. W. 1996. Molecular markers reveal cryptic sex in the human pathogen Coccidioides immitis. Proc. Natl. Acad. Sci. USA 93:770-773.

Cao, S., Zhang, B., Li, M., Xu, S., Luo, H., Jin, S., Jia, Q., Huang, J., Jin, M., and $\mathrm{Wu}, \mathrm{S}$. 2011. Postulation of stripe rust resistance genes and analysis of adult in 50 wheat varieties (lines) in Gansu Province. (in Chinese with English abstract). Acta Agron. Sin. 37:1360-1371.

Chen, W., Wu, L., Liu, T., Xu, S., Jin, S., Peng, Y., and Wang, B. 2009. Race dynamics, diversity, and virulence evolution in Puccinia striiformis $\mathrm{f}$. sp. tritici, the causal agent of wheat stripe rust in China from 2003 to 2007. Plant Dis. 93:1093-1101.

Chen, W., Xu, S., Liu, T., Lin, R., Wu, L., Jin, S., and Cao, S. 2007. Wheat stripe rust and its prospects for ecological control in China. Proceedings of 16th International Plant Protection Congress. Glasgow 2:11D.

Chen, X., Line, R. F., and Leung, H. 1993. Relationship between virulence variation and DNA polymorphism in Puccinia striiformis. Phytopathology 83:1489-1497.
Chowdhary, A., Hiremath, S. S., Sun, S., Kowshik, T., Randhawa, H. S., and $\mathrm{Xu}, \mathrm{J} .2011$. Genetic differentiation, recombination and clonal expansion in environmental populations of Cryptococcus gattii in India. Environ. Microbiol. 13:1875-1888.

Enjalbert, J., Duan, X., Giraud, T., Vautrin, D., de Vallavieille-Pope, C., and Solignac, M. 2002. Isolation of twelve microsatellite loci, using an enrichment protocol, in the phytopathogenic fungus Puccinia striiformis f. sp. tritici. Mol. Ecol. Notes 2:563-565.

Evanno, G., Regnaut, S., and Goudet, J. 2005. Detecting the number of clusters of individuals using the software STRUCTURE: A simulation study. Mol. Ecol. 14:2611-2620.

Gao, H., Williamson, S., and Bustamante, C. D. 2007. A Markov Chain Monte Carlo approach for joint inference of population structure and inbreeding rates from multilocus genotype data. Genetics 176:1635-1651.

Hovmøller, M. S., Yahyaoui, A. H., Milus, E. A., and Justesen, A. F. 2008. Rapid global spread of two aggressive strains of a wheat rust fungus. Mol. Ecol. 17:3818-3826.

Justesen, A. F., Ridout, C. J., and Hovmøller, M. S. 2002. The recent history of Puccinia striiformis f. sp. tritici in Denmark as revealed by disease incidence and AFLP markers. Plant Pathol. 51:13-23.

Li, Z., and Zeng, S. 2002. Wheat Rusts in China. China Agricultural Publisher, Beijing.

Liang, J., Wan, Q., Luo, Y., and Ma, Z. 2013. Population genetic structures of Puccinia striiformis in Ningxia and Gansu Provinces of China. Plant Dis. 97:501-509.

Liu, X., Huang, C., Sun, Z., Liang, J., Luo, Y., and Ma, Z. 2011. Analysis of population structure of Puccinia striiformis in Yunnan Province of China by using AFLP. Eur. J. Plant Pathol. 129:43-55.

Lu, N., Wang, J., Chen, X., Zhan, G., Chen, C., Huang, L., and Kang, Z. 2011. Spatial genetic diversity and interregional spread of Puccinia striiformis $\mathrm{f}$. sp. tritici in Northwest China. Eur. J. Plant Pathol. 131:685-693.

Mboup, M., Leconte, M., Gautier, A., Wan, A. M., Chen, W., de Vallavieille-Pope, C., and Enjalbert, J. 2009. Evidence of genetic recombination in wheat yellow rust populations of a Chinese oversummering area. Fungal Genet. Biol. 46:299-307.

McDonald, B. A., and Linde, C. 2002. Pathogen population genetics, evolutionary potential and durable resistance. Annu. Rev. Phytopathol. 40: 349-379.

Nei, M. 1972. Genetic distance between populations. Am. Nat. 106:283-292.

Parks, J. C., and Werth, C. R. 1993. A study of spatial features of clones in a population of bracken fern, Pteridium aquilinum (Dennstaedtiaceae). Am. J. Bot. 80:537-544.

Peakall, R., and Smouse, P. E. 2006. GENALEX 6, genetic analysis in Excel. Population genetic software for teaching and research. Mol. Ecol. Notes 6: 288-295.

Pritchard, J. K., Stephens, M., and Donnelly, P. 2000. Inference of population structure using multilocus genotype data. Genetics 155:945-959.

Shi, S. 2004. Climate-based regional classification for oversummering and overwintering of Puccinia striiformis and spatio-temporal dynamic analysis in China with GIS. Dissertation. China Agricultural University, Beijing.

Singh, R. P., William, H. M., Huerta-Espino, J., and Rosewarne, G. 2004. Wheat rust in Asia: Meeting the challenges with old and new technologies. Proceedings of the $4^{\text {th }}$ International Crop Science Congress, Brisbane, Australia. http://www.cropscience.org.au/icsc2004/symposia/3/7/141_singhrp.htm\# TopOfPage

Steele, K. A., Humphreys, E., Wellings, C. R., and Dickinson, M. J. 2001. Support for a stepwise mutation model for pathogen evolution in Australasian Puccinia striiformis f. sp. tritici by use of molecular markers. Plant Pathol. 50:174-180.

Stubbs, R. W. 1985. Stripe rust. Pages 61-101 in: The Cereal Rusts. A. P. Roelfs and W. R. Bushnell, eds. Academic Press, New York.

Swofford, D. L. 1998. PAUP, Phylogenetic Analysis Using Parsimony (and Other Methods). Sinauer Associates Press, Sunderland, MA.

Vos, P., Hogers, R., Bleeker, M., Reijans, M., Van de Lee, T., Hornes, M., Frijters, A., Pot, J., Peleman, J., Kuiper, M., and Zebeau, M. 1995. AFLP, a new concept for DNA finger printing. Nucleic Acids Res. 23: 4407-4414.

Wan, A., Chen, X., and He, Z. 2007. Wheat stripe rust in China. Crop Pasture Sci. 58:605-619.

Wan, A., Zhao, Z., Chen, X., He, Z., Jin, S., Jia, Q., Yao, G., Yang, J., and Wang, B. 2004. Wheat stripe rust epidemic and virulence of Puccinia striiformis f. sp. tritici in China in 2002. Plant Dis. 88:896-904.

Wang, J., Chen, C., Lu, N., Peng, Y., Shan, G., Huang, L., and Kang, Z. 2010. SSR analysis of population genetic diversity of Puccinia striiformis $\mathrm{f}$. sp. tritici in Sichuan Province, China. (in Chinese with English abstract) Mycosystem 29:206-213.

Xia, X., Yao, G., Liu, Z., and Niu, Y. 2005. Population of resistance genes to stripe rust in common wheat cultivars from Sichuan Province. (in Chinese with English abstract) Xi Nan Nong Ye Xue Bao 18:422-426. 
Xie, S., Chen, Y., and Chen, W. 1998. Occurrence and epidemics of wheat stripe rust in Abazhou. (in Chinese with English abstract) Acta Phytophysiol. Sin. 15:85-91.

Yang, H., and Stubbs, R. W. 1990. Gene postulation for wheat stripe rust resistance on Chinese differential hosts. (in Chinese with English abstract) Scientia Agric. Sin. 17:67-72.
Yeh, F. C., Yang, R. C., Boyle, T. B., Ye, Z. H., and Mao, J. X. 1997. POPGENE, the user-friendly shareware for population genetic analysis. Molecular Biology and Biotechnology Centre, University of Alberta, Canada.

Zeng, S., and Luo, Y. 2006. Long-distance spread and interregional epidemics of wheat stripe rust in China. Plant Dis. 90:980-988. 